\title{
A numerical simulation for reduction of rudder cavitation with gap flow blocking bars
}

\author{
Jungkeun $\mathrm{Oh}^{1}$, Seung-Hee Lee ${ }^{2}$ Sang-Hyun $\mathrm{Kim}^{2}$ and Dae-Won Seo ${ }^{1}$ \\ ${ }^{1}$ Jungseok Research Institute of International Logistics and Trade, Inha University, Korea \\ ${ }^{2}$ Department of Naval Architecture \& Ocean Engineering, Inha University, Korea
}

\begin{abstract}
In recent practices, a half circular prismatic bar protruding beyond the concave surface of the horn facing the gap has been formed along the centerplane of a rudder to lessen the gap flow between the horn and the movable portion of the rudder system. If a flow through the gap of a rudder is reduced considerably through this approach, previous numerical studies indicate that not only the gap flow but also the rudder cavitation can be noticeably diminished. In the present study, numerical simulations on two-dimensional rudder sections were performed to show that the blocking ability of the single centre bar can be improved by the proper choice of sectional shape. Moreover, a pair of blocking bars attached symmetric to the centerplane on the opposite convex surface of the movable portion is suggested in the study as well, to circumvent the difficulties arising from the practical application of the single centre bars. The bars are placed near the outer edges of the gap easily accessible at the maximum rudder angle to allow simple installation of the device during a maintenance period of a ship. It is found that the pair of blocking bars further improves the blocking effects and application to a practical three-dimensional rudder also backs up the fact.
\end{abstract}

KEY WORDS: Rudder cavitation; Blocking bar; Gap flow.

\section{INTRODUCTION}

Rudder cavitation is frequently observed in large container ships running fast and may cause serious damage to the rudder devices of the ship, and consequently can be detrimental to the ship safety and cost-effectiveness. Rudder cavitation occurs more frequently and is more severely when a ship is cruising straight at high speed, rather than maneuvering with a large rudder angle, since the ship operates mostly in the former stage. Rudder cavitation occurs a lot in the vicinity of two regions: the leading edge of the rudder, and the gap between the fixed (horn and pintle) and the movable portion. The former cavitation can usually be mitigated by modifying the rudder section (Kim, Lee and Byun, 2008; Shen, Jiang and Remmers, 1997; Park and Lee, 2010) and the latter by reducing the gap clearance. A substantial reduction in gap clearance can raise serious technical difficulties in the rudder installation processes and studies have been done to moderate the gap cavitation by other means. Rhee and Kim (2008) suggested a cam device for gap flow blocking and Paik, et al.(2008), Rhee, Lee, Lee and Oh (2010), and Seo, Lee, Kim and Oh (2012) demonstrated that the device can suppress the gap cavitation through model experiments and computations. Seo, Lee, Oh and Kim (2010) showed that the jet blowing system devised for a high-lift rudder can be utilized in order to mitigate the gap cavitation of a rudder with a small amount of flow injection in the reverse direction. The device, however, cannot be easily installed in the rudder system of a ship, and is more difficult if the ship is already in service. Boo, Song and Shin (2004) investigated the characteristics of rudder cavitation at various Reynolds numbers and examined the effectiveness of a half round shaped blocking bar placed at the middle of the gap. He found that modest reductions in gap

Corresponding author: Dae-Won Seo

e-mail: dwseo@inha.ac.kr 
cavitation are achievable with the device but it is also hard to apply to an existing rudder system.

In the present study, it is shown that the blocking ability of the aforementioned single bar can be enhanced with the proper choice of cross sectional shapes. However, to circumvent the difficulties in implementation, a pair of blocking bars symmetric to the centerplane and attached on the convex surface of the movable portion facing the gap is devised to improve the blocking performance. The bars are placed near the outer edges of the gap easily accessible at the maximum rudder angle to allow simple installation of the device during routine maintenance periods of a ship. Numerical simulations have been performed to show that the use of a pair instead of single blocking bar further improves the blocking effects. The new blocking bars are installed in a realistic 3-dimensional horn-type rudder system and performance of the device is examined through numerical computations to ensure its practicability.

\section{COMPUTATIONAL ARRANGEMENTS}

For numerical study of the performance of the devices for blocking gap flow, a scale model of a horn-type rudder of an 8,000 TEU class container ship with a design speed of 25 knots was selected as shown in Fig. 1 . The scale ratio of the model to the original rudder was chosen to be $1 / 10$ and the effects of the wake behind a propeller and hull were not considered.

In the initial stage, two-dimensional computations were performed to investigate the influence of cross sectional shapes upon the effectiveness of the blocking bars. Two sections were selected at the horn and pintle of the rudder model for 2 dimensional analyses and Fig. 1 shows the sections. The chord lengths of each section (horn/pintle section) are $0.8 \mathrm{~m}$ and $0.746 \mathrm{~m}$, respectively and the gap clearances of the sections are identically $5 \mathrm{~mm}$. The computation was conducted at the rudder angle of $3^{\circ}$ since the rudder angle of a ship is usually within $\pm 3^{\circ}$ when cruising straight.

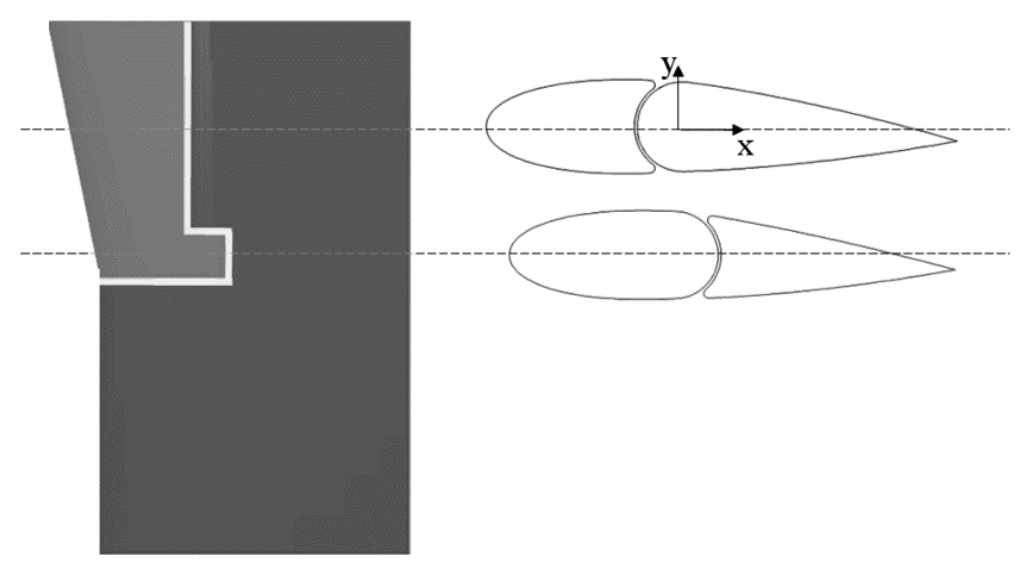

Fig. 1 Profile of a horn type rudder of an 8,000 TEU class container ship and numerical models for two dimensional computations; a horn section and a pintle section.

As shown in Fig. 2, the numerical grids for two-dimensional computations were chosen to have C-type topologies in the Cartesian coordinates $(x, y)$ where positive $x$ is taken parallel to the incoming flow direction. The calculation domains in $x$ - and $y$-axis range $-2.6 \leqq x / c \leqq 3.6$ and $-2.6 \leqq y / c \leqq 2.6$, respectively, based on the chord lengths $c$ of the each section. A total numbers of 45,000 grid points were used for the calculation domain, and 21 grid lines were placed across the gap between the horn and the rudder surfaces and $Y^{+}$has been confined to not exceed 65 .

After that, computations were carried out to study the flow characteristics around the three-dimensional rudder model equipped with the pair of blocking bars found to be the most effective among the two-dimensional models tested and the results were compared with the case without any gap flow blocking device. The numerical grid for the three-dimensional horn-type rudder system is shown in Fig. 3. The grid has a C-H type topology, and the computation ranges were chosen to be $-2.6 \leqq x / c \leqq$ $3.6,-2.6 \leqq y / c \leqq 2.6$, and $0 \leqq z / c \leqq 1$ based on the chord length of $0.746 \mathrm{~m}$, the same as the two-dimensional pintle section. A total numbers of 1,700,000 grid points were used where the numbers of grid points on each side of the rudder surface were $75 \times$ 66. $15 \times 35$ gird points and $15 \times 25$ girds point were used on the bar surface in the horn and pintle part respectively. In addition, 21 grid lines were placed across the gap between the horn and the rudder surfaces and $Y^{+}$has been confined not to exceed 150 . 

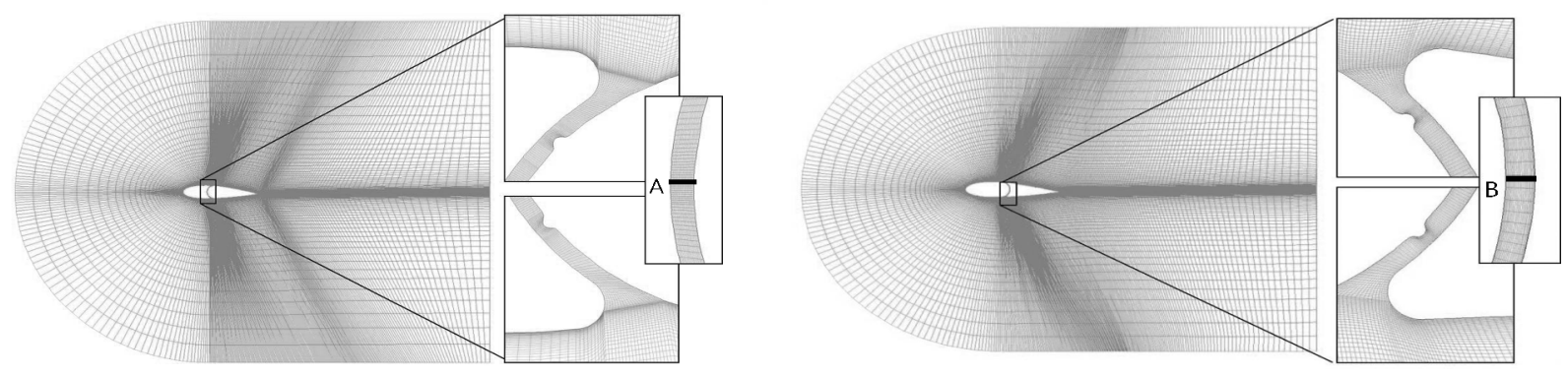

Fig. 2 Computational domains and grid around the horn and pintle sections.

Throughout the present study, a commercial code, FLEUNT v6.3 has been used for numerical computations, which employs a cell-centered finite-volume method along with a linear reconstruction scheme in order to use computational cells of arbitrary polyhedral shapes. A SIMPLE type segregated algorithm was chosen for the velocity-pressure coupling. The discretization schemes for pressure and momentum were 2nd order accurate and the turbulent kinetic energy and dissipation rate were discretized by QUICK and 1st order upwind scheme to include the complex flow near the gap in the 2D calculations. In general, the gap flow which spurts out from the gap of the rudder section meets a rapid change of area and pressure nearby the way out of the gap so that makes complicated flow. In the zone mentioned above, much turbulent kinetic energy is produced and the dissipation quantity is small (Guo, Langrish and Fletcher, 2001). In other words it is important to presume the kinetic energy on the free shear layer, so we used a high-order scheme on the kinetic energy.

In cases of 3D, a SIMPLE type segregated algorithm was also chosen for the velocity-pressure coupling. The discretization schemes for pressure and momentum were 2 nd order accurate as the same of $2 \mathrm{D}$ calculations and turbulent kinetic energy and dissipation rate were discretized by 1 st order upwind scheme and time derivatives by a 1st order backward implicit scheme to enhance the stability of convergence and calculating times.

The discretized equations were solved using point wise Gauss-Seidel iterations, while the algebraic multi-grid method accelerates the solution convergence. The relaxation factors were set to be 0.3 for the pressure, 0.7 for the momentum, and 0.8 for the turbulence through the present calculations. The turbulence of the flow was approximated by Realizable $k-\varepsilon$ with a standard wall function. Boundary conditions were defined to have (1) uniform flow with $6 \mathrm{~m} / \mathrm{s}$ speed $(u=1, v=w=0)$ at the inlet boundary of the domain and (2) static pressure at the outer boundary of the domain. The speed of the uniform flow has been determined to be $6 \mathrm{~m} / \mathrm{s}$ based on the result of Seo, Lee, Oh and Kim (2010) which did not include the effects of the wake behind a propeller and hull.

Mass flow rate, which is the value to tell the gap cavitation strength indirectly, can be calculated by $\dot{m}=\rho \mathrm{v} \cdot A$, where v is the flow velocity passing through the gap. The mass flow rate $(\dot{m})$ was computed at locations $A$ and $B$ as shown in Fig. 2 .

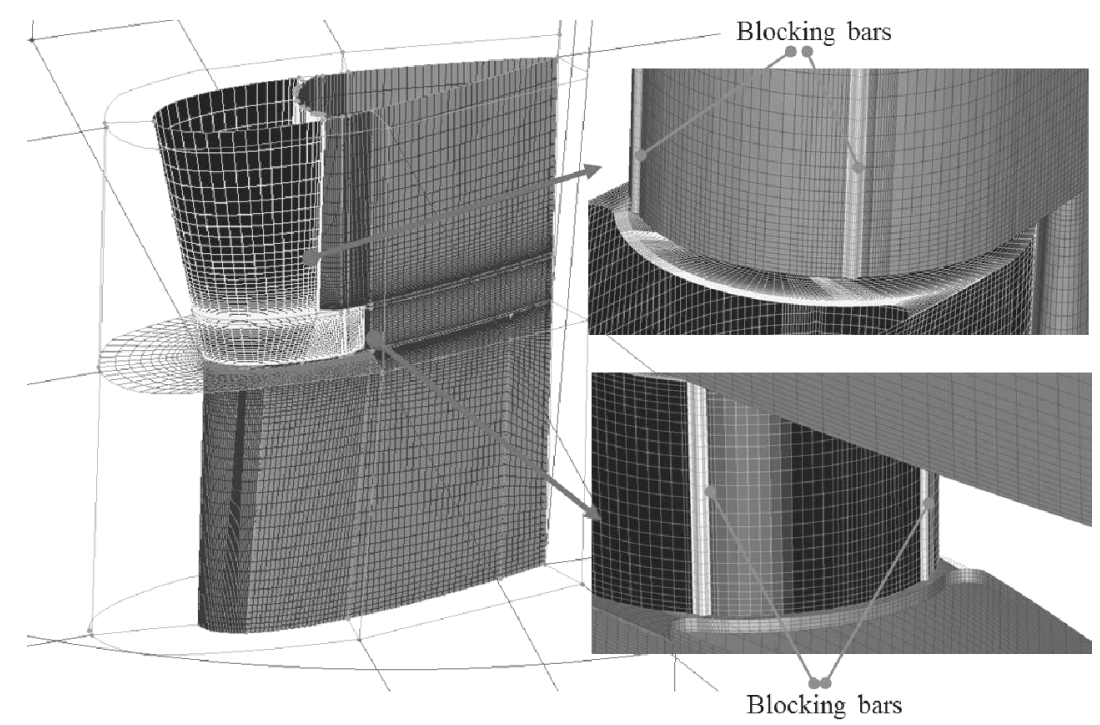

Fig. 3 Three-dimensional numerical grids around the horn type rudder model. 


\section{RESULTS AND DISCUSSION}

\section{Two dimensional gap flows}

\section{Gap flow without a blocking bar}

Numerical computations for the simulation of flow characteristics around the gap of the horn and pintle sections absent of a blocking device were conducted for comparison. The results are shown in Fig. 4 where pressure distribution around the gap is given. Table 1 shows the mass flow rate through the gap of the two sections; $21.7 \mathrm{~kg} / \mathrm{s}$ for the horn portion section and $23.5 \mathrm{~kg} / \mathrm{s}$ for the pintle. The Figure also indicates that, for both sections, cavitation is plausible near the gap of the suction side of the movable portion and near the pressure side of the fixed portion of horn section.

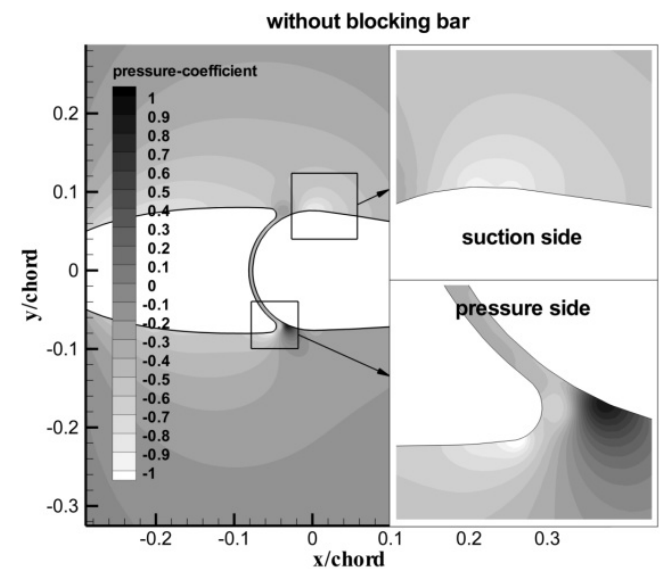

(a) Horn section.

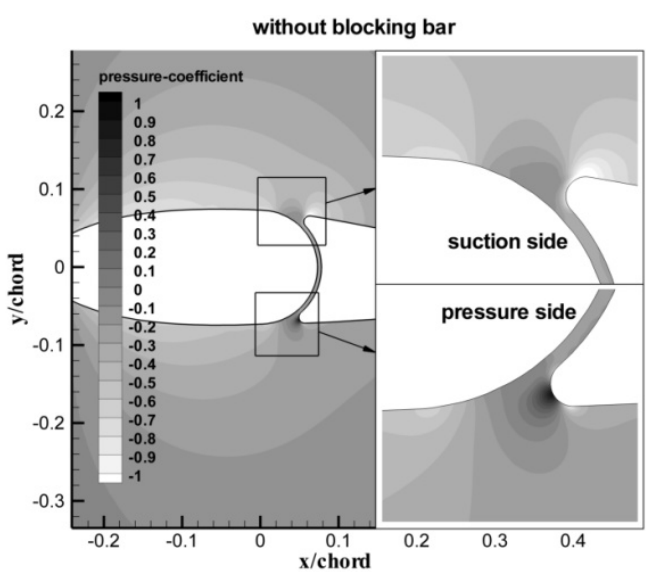

(b) Pintle section.

Fig. 4 Pressure distribution around the horn and pintle section $\left(R e=4.5 \times 10^{6}\right.$, rudder angle $=3^{\circ}$, flow speed $\left.=6 \mathrm{~m} / \mathrm{s}\right)$.

Table 1 Flux per unit area of ordinary rudder section without blocking bar

$\left(R e=4.5 \times 10^{6}\right.$, rudder angle $=3^{\circ}$, flow speed $\left.=6 \mathrm{~m} / \mathrm{s}\right)$.

\begin{tabular}{|l|c|c|}
\hline & Horn section & Pintle section \\
\hline Flow rate $(\mathrm{kg} / \mathrm{s})$ & 21.7 & 23.5 \\
\hline Minimum of $C_{p}$ & -1.189 & -1.586 \\
\hline
\end{tabular}

\section{Gap flow retardation with blocking bars}

The gap between the fixed and movable portion of the rudder is necessary to permit allowance inevitable needed for assembly and operation of the rudder system. The gap clearance varies with the size of the rudder and the accuracies in manufacturing processes. Rudder gap clearances of large ships under consideration in the present study vary between $50 \sim 100$ $m m$. It is well known that gap flow is one of the main sources of rudder cavitation. However, it is not possible to block the gap flow completely even with the blocking bars since the gap clearance varies with rudder angle and oversized bars may cause jamming of the rudder during operation. Accordingly, it is a common practice that blocking bars of a half-circular cylindrical shape not higher than 0.4 times the gap clearance are fillet jointed on the concave surface of a fixed portion facing the gap in the casting stage of horns.

Numerical computations were done to investigate the effect of a fillet jointed blocking bar with a half-circular sectional shape. The bar was attached to the surface of the horn section and the computed results are shown in Fig. 5. The left figure shows the sectional shape of the blocking bar and the right the pressure distribution. The figure shows that the low pressure region marked with a white color is slightly reduced but still notable even after installation of the bar. Table 2 also gives that the mass flow rate for the case has been reduced to $18.4 \mathrm{~kg} / \mathrm{s}$, a $15.2 \%$ reduction compared to the results of the unblocked case, but the results indicate on the whole that further improvement in blocking ability is necessary to suppress the gap cavitation significantly by the attachment of blocking bars. 

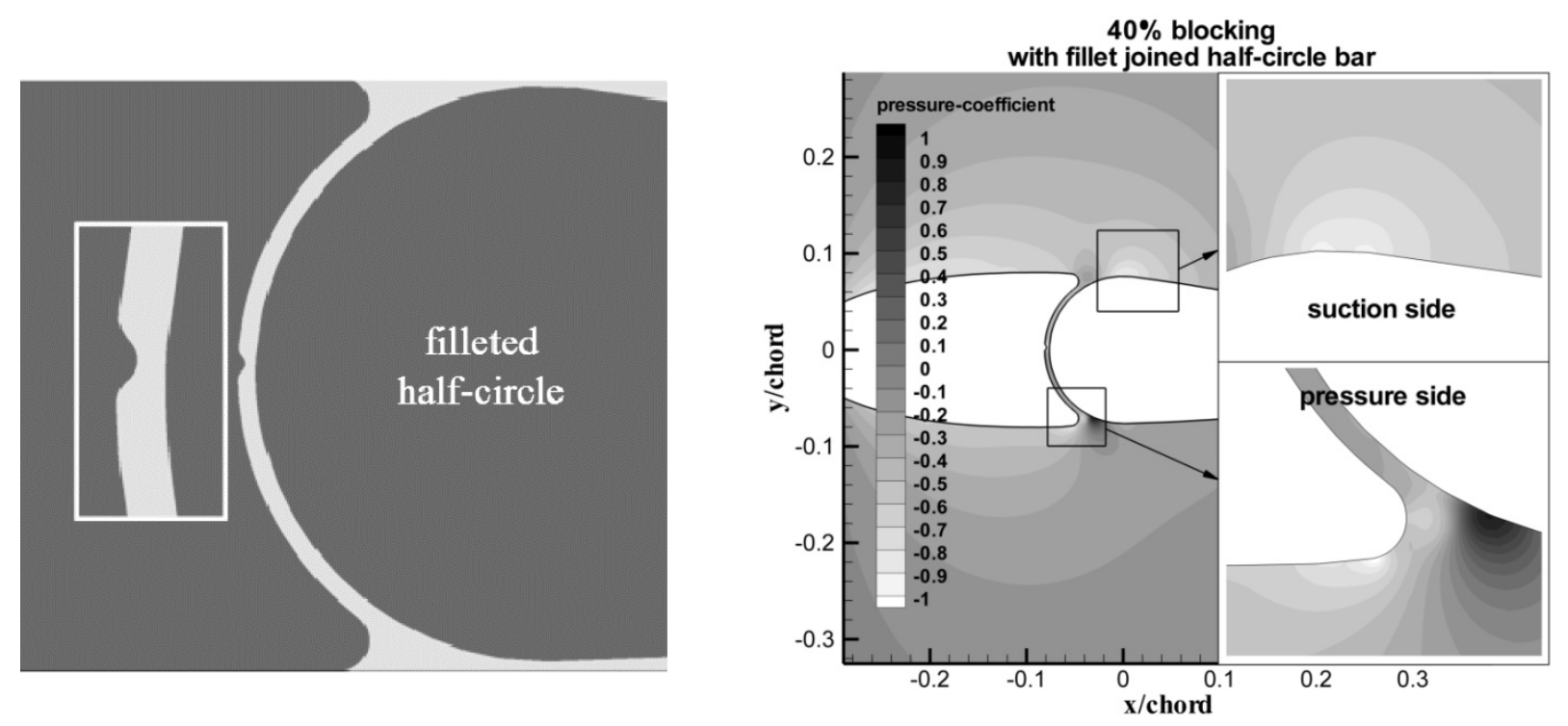

Fig. 5 Fillet jointed half-circular cylindrical bar and resulting pressure distribution around the gap (horn section, $R e=4.5 \times 10^{6}$, rudder angle $=3^{\circ}$, flow speed $=6 \mathrm{~m} / \mathrm{s}$ ).

Table 2 Blocking of gap flux per unit area with the fillet jointed half-circular cylindrical bar (horn section, $R e=4.5 \times 10^{6}$, rudder angle $=3^{\circ}$, flow speed $=6 \mathrm{~m} / \mathrm{s}$ ).

\begin{tabular}{|c|c|c|}
\hline Bar protrusion (\%) & Flow rate $(\mathrm{kg} / \mathrm{s})$ & Reduction (\%) \\
\hline 40 & 18.4 & 15.2 \\
\hline
\end{tabular}

To investigate the influence of sectional shapes of the blocking bars, three additional sections for the bar, namely, rectangular, half-circular and smooth sectional shapes have been tested as shown in Fig. 6 . The height of the bars were fixed at $40 \%$ of the gap clearance and the blocking effects were compared each other.

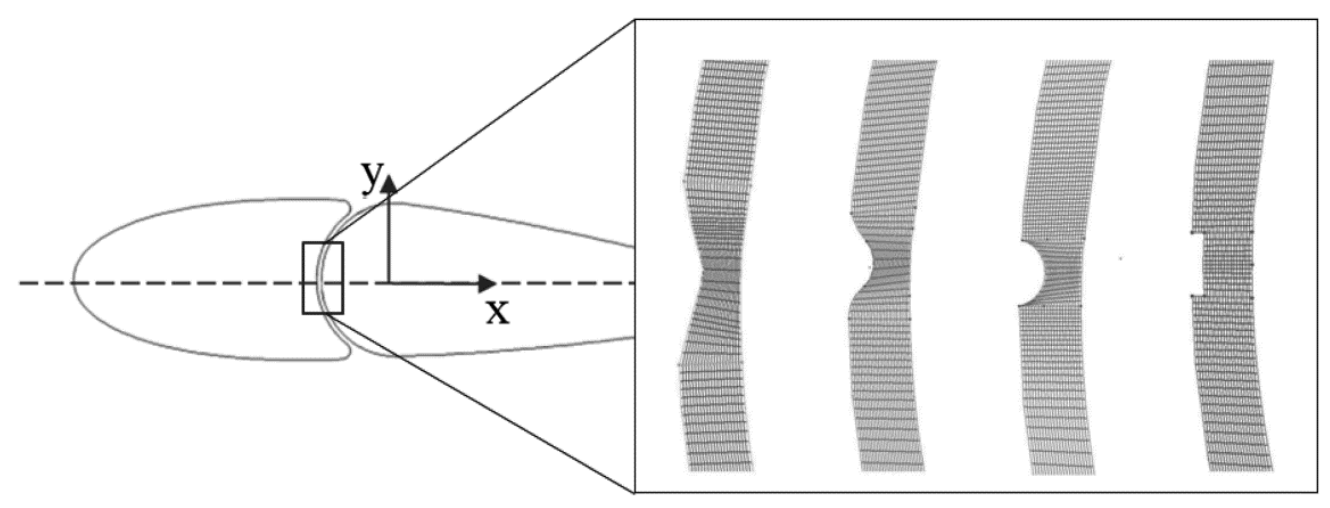

Fig. 6 Various sectional shapes of two-dimensional blocking bars.

The pressure distributions near the gap corresponding to the bars with different sectional shapes are shown in Fig. 7. Table 3 shows the variation in reduction rates of the gap flow due to the difference in sectional shapes. From the results, it is found that the bar with the rectangular sectional shape evoking the swiftest change in the gap clearance blocks the gap flow most efficiently as well. Employment of the blocking bars with a rectangular sectional shape yields more than $40 \%$ reduction of the gap flow, about 30\% improvement over the conventional fillet jointed half-circular cylindrical bar. 
Table 3 Comparison of gap flux per unit area and reduction rates of the bars with various cross sectional shapes (horn section, $R e=4.5 \times 10^{6}$, rudder angle $=3^{\circ}$, flow speed $=6 \mathrm{~m} / \mathrm{s}$, Horn section, protrusion height $40 \%$ ).

\begin{tabular}{|c|c|c|c|}
\hline Sectional shape & Flow rate $(\mathrm{kg} / \mathrm{s})$ & Reduction $(\%)$ & Minimum of $C_{p}$ \\
\hline Smooth & 19.8 & 8.8 & -1.107 \\
\hline Fillet jointed half-circular & 18.4 & 15.2 & -1.103 \\
\hline Half-circular & 17.8 & 18.0 & -1.034 \\
\hline Rectangular & 12.7 & 41.5 & -0.758 \\
\hline
\end{tabular}

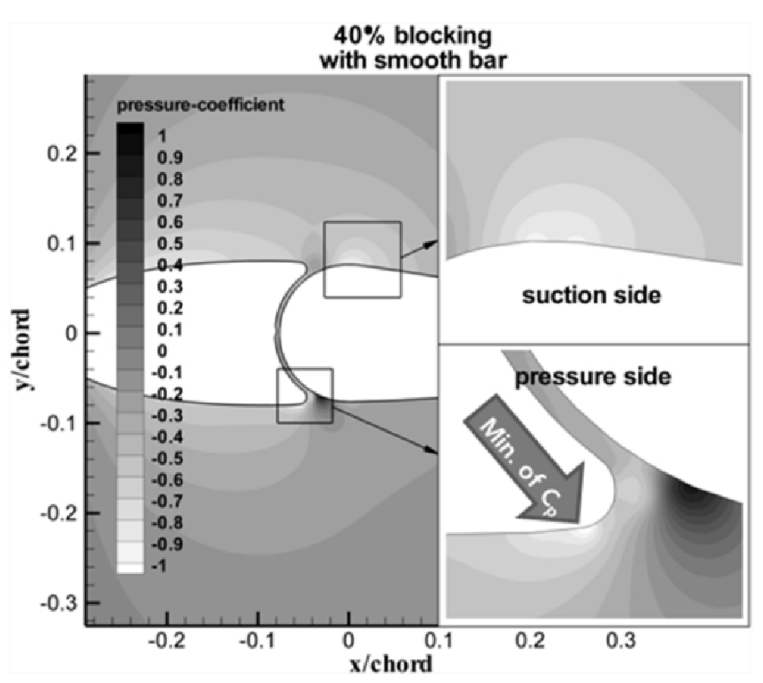

(a) Smooth.

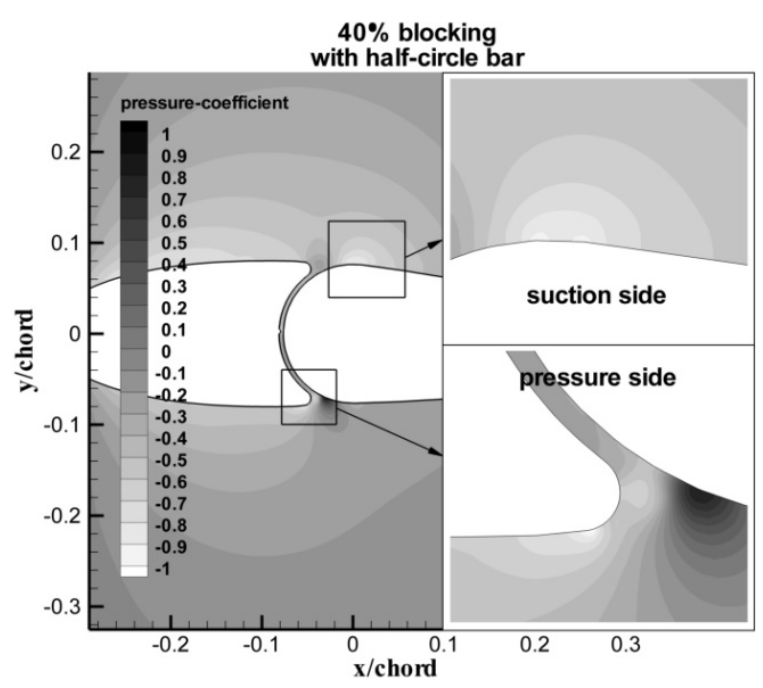

(b) Half-circular.

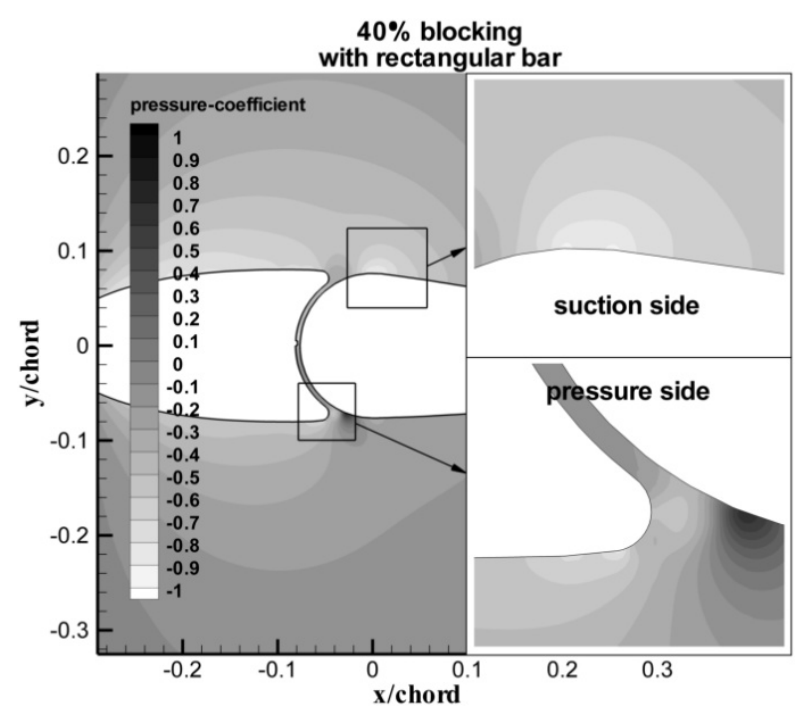

(c) Rectangular.

Fig. 7 Pressure distributions around the gap of the horn section for various sectional shapes of the blocking bar $\left(R e=4.5 \times 10^{6}\right.$, rudder angle $=3^{\circ}$, flow speed $\left.=6 \mathrm{~m} / \mathrm{s}\right)$.

\section{Gap flow variation with the blocking bar heights}

It is presumable that a higher bar may block the gap flow more efficiently and hence the influence of the blocking bar heights on the reduction of the gap flow has been studied to validate the fact. The sectional shape of the bar for checking this presumption was chosen to be rectangular, as it was already found to be the most effective in gap flow retardation among the 
sectional shapes tested. In Fig. 8, the changes in the pressure distribution with the variation of the bar heights are shown and the corresponding blocking effects are summarized in Table 4. As expected it was found that a higher blocking bar can retard the gap flow more effectively. Only the practical limitation encountered in the implementation of blocking bars may restrict the heights of blocking bars, since it is necessary to avoid any interference between the fixed and movable portion of the rudder during installation and operation as well.

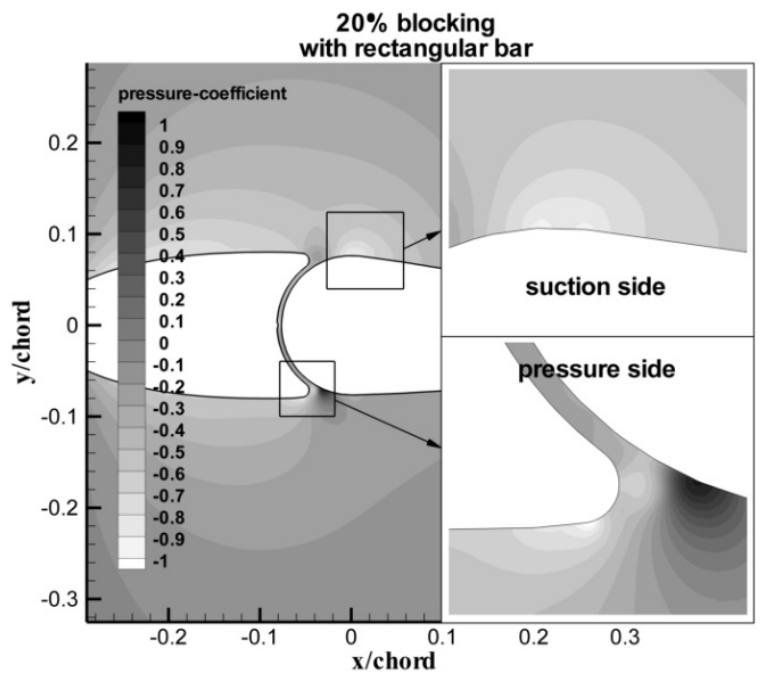

(a) $20 \%$ blocking.

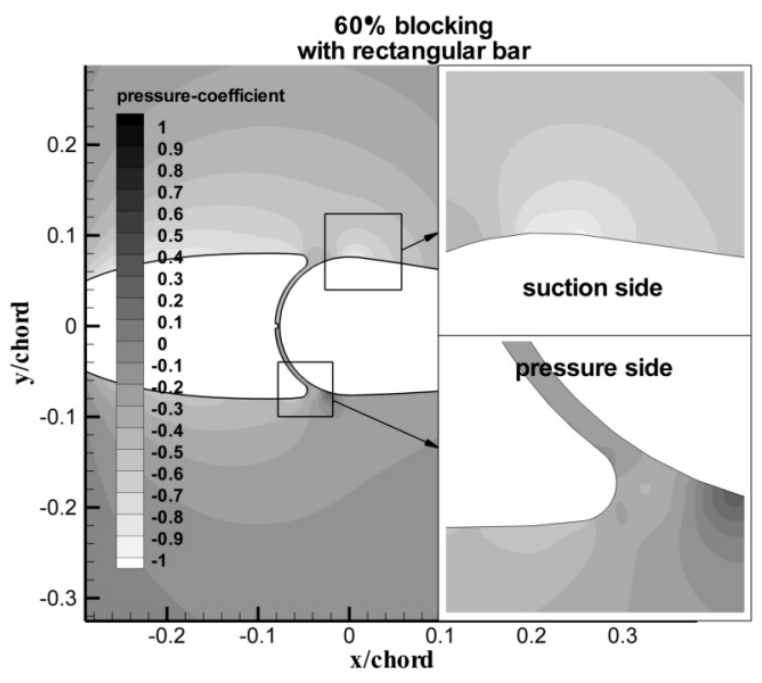

(b) $60 \%$ blocking.

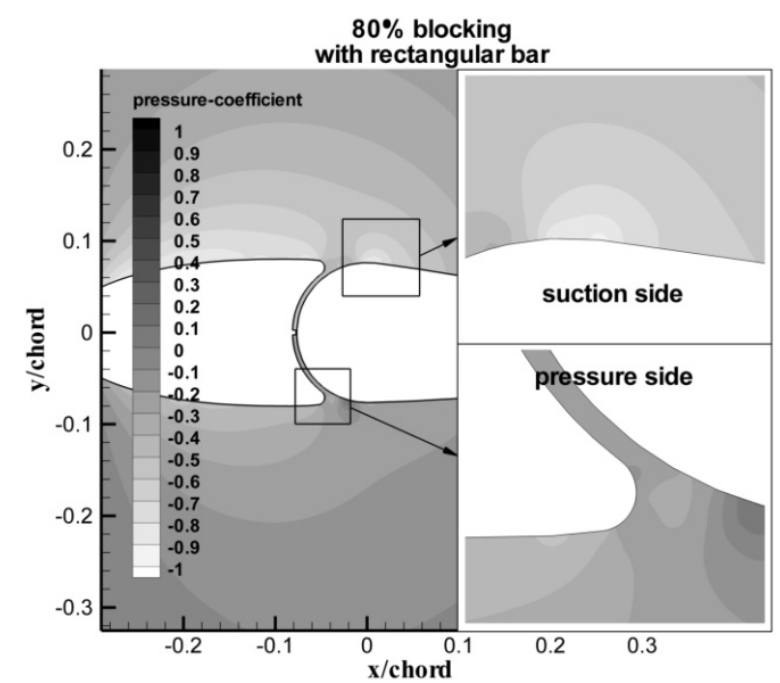

(c) $80 \%$ blocking.

Fig. 8 Pressure distributions around the horn section with various heights of rectangular blocking bar $\left(R e=4.5 \times 10^{6}\right.$, rudder angle $=3^{\circ}$, flow speed $\left.=6 \mathrm{~m} / \mathrm{s}\right)$.

Table 4 Influence of bar height on the gap flux per unit area and the reduction rates

$\left(R e=4.5 \times 10^{6}\right.$, rudder angle $=3^{\circ}$, flow speed $=6 \mathrm{~m} / \mathrm{s}$, Horn section, rectangular shape $)$.

\begin{tabular}{|c|c|c|c|}
\hline Height (\%) & Flow rate $(\mathrm{kg} / \mathrm{s})$ & Reduction $(\%)$ & Minimum of $C_{p}$ \\
\hline 20 & 19.6 & 9.7 & -0.965 \\
\hline 40 & 12.7 & 41.9 & -0.758 \\
\hline 60 & 6.05 & 72.1 & -0.552 \\
\hline 80 & 2.75 & 87.3 & -0.482 \\
\hline
\end{tabular}




\section{The effect of two blocking bars attached symmetric to the centerplane}

Previously in the present study, it was found that a bar with a rectangular cross section can block the gap flow most effecttively from among the shapes tested and the effect becomes more prominent as the bar becomes higher. However, it's not easy in practice to attach a rectangular bar on the surface of the fixed portion in the casting process and the viability becomes even worse if trying to use higher ones since then possibility of interference with the movable portion will be also increased.

For the purpose of obtaining as much efficiency in retarding the gap flow as rectangular bars and to make it easy to install inside the rudder gap without worrying about interferences, a pair of blocking bars of half circular shape fillet jointed symmetric to the centerplane (symmetric bars, hereafter) instead of one has been suggested in the present study. Especially, the bars are devised to be easily attached on the rudder system at the maximum angle, usually at $35^{\circ}$, and so it can be installed on the rudder of a ship already in operation when docking for routine maintenance. Furthermore, rudder cavitation usually occurs near the cruising speed of a ship at which rudder angles are kept sufficiently small and will not damage the bar's ability to efficiently block the gap flow. Fig. 9 shows the location of the symmetric bars. The bars protrude from the convex surfaces of the gap at the points located $30^{\circ}$ or $40^{\circ}$ apart from the centerline of the movable and fixed portions, marked with dotted lines in the figure, to keep the bars inside the gap when cruising with small rudder angles but to expose them out of the gap at the maximum rudder angles.

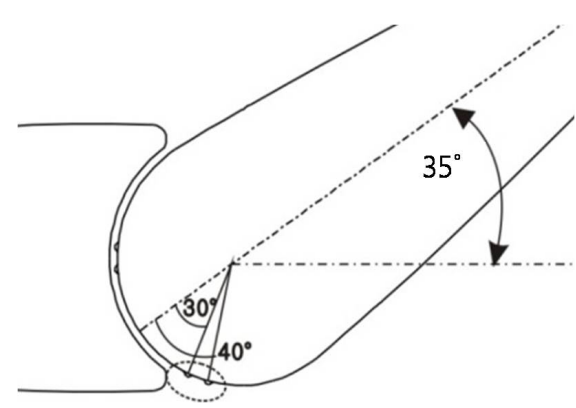

(a) Horn section.

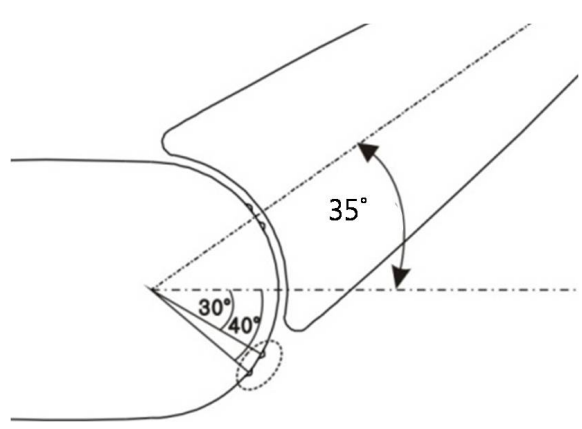

(b) Pintle section.

Fig. 9 Location of symmetric blocking bars installed inside the rudder gap.

The results of numerical computation for various locations of the symmetric blocking bars are summarized in Table 5. Fig. 10 and Fig. 11 show the pressure distributions around the horn and pintle section, respectively.

Table 5 summarizes the computed results of the reduction in gap flow with the employment of the symmetric bars attached at $\pm 30^{\circ}$ and $\pm 40^{\circ}$ from the centerline. At the horn section, reduction rates of gap flow with symmetric bars are 35.9 and $41.5 \%$ at the angles of $\pm 30^{\circ}$ and $\pm 40^{\circ}$, respectively. At the pintle section, the symmetric bars show better reduction rates of 37.5 and $52.0 \%$, respectively. According to Table 3, the best reduction rate achievable with a single center bar is $41.5 \%$ if a rectangular sectional shape is used. The reduction rate of $41.5 \%$ attained at the horn section with the symmetric bar is identical to that amount and $52.0 \%$ at the pintle section is almost $30 \%$ improvement from the efficiency of a single rectangular blocking bar. It can be concluded that the symmetric blocking bars placed inside the gap of the pintle section at $\pm 40^{\circ}$ with the respect to the centerline is the most effective one among various two-dimensional sections tested.

Table 5 Effect of the gap flow blocking bars symmetrically installed at various locations $\left(R e=4.5 \times 10^{6}\right.$, rudder angle $=3^{\circ}$, flow speed $\left.=6 \mathrm{~m} / \mathrm{s}\right)$.

\begin{tabular}{|c|c|c|c|c|}
\hline Install surface & Location (from CL) & Flow rate $(\mathrm{kg} / \mathrm{s})$ & Reduction (\%) & Minimum of $C_{p}$ \\
\hline \multirow{3}{*}{ Horn section } & $\pm 30^{\circ}$ & 13.9 & 35.9 & -0.758 \\
\cline { 2 - 5 } & $\pm 40^{\circ}$ & 12.7 & 41.5 & -0.620 \\
\hline \multirow{3}{*}{ Pintle section } & $\pm 30^{\circ}$ & 14.7 & 37.5 & -1.27 \\
\cline { 2 - 5 } & $\pm 40^{\circ}$ & 11.3 & 52.0 & -1.17 \\
\hline
\end{tabular}


Fig. 10 and Fig. 11 also indicate that the gap flow is considerably retarded with the employment of the symmetric bars for both the horn and pintle sections. In addition, if the performance of a single center bar is not satisfactory, extra symmetric bars may be added to block up the gap flow without any interference with the existing bar.
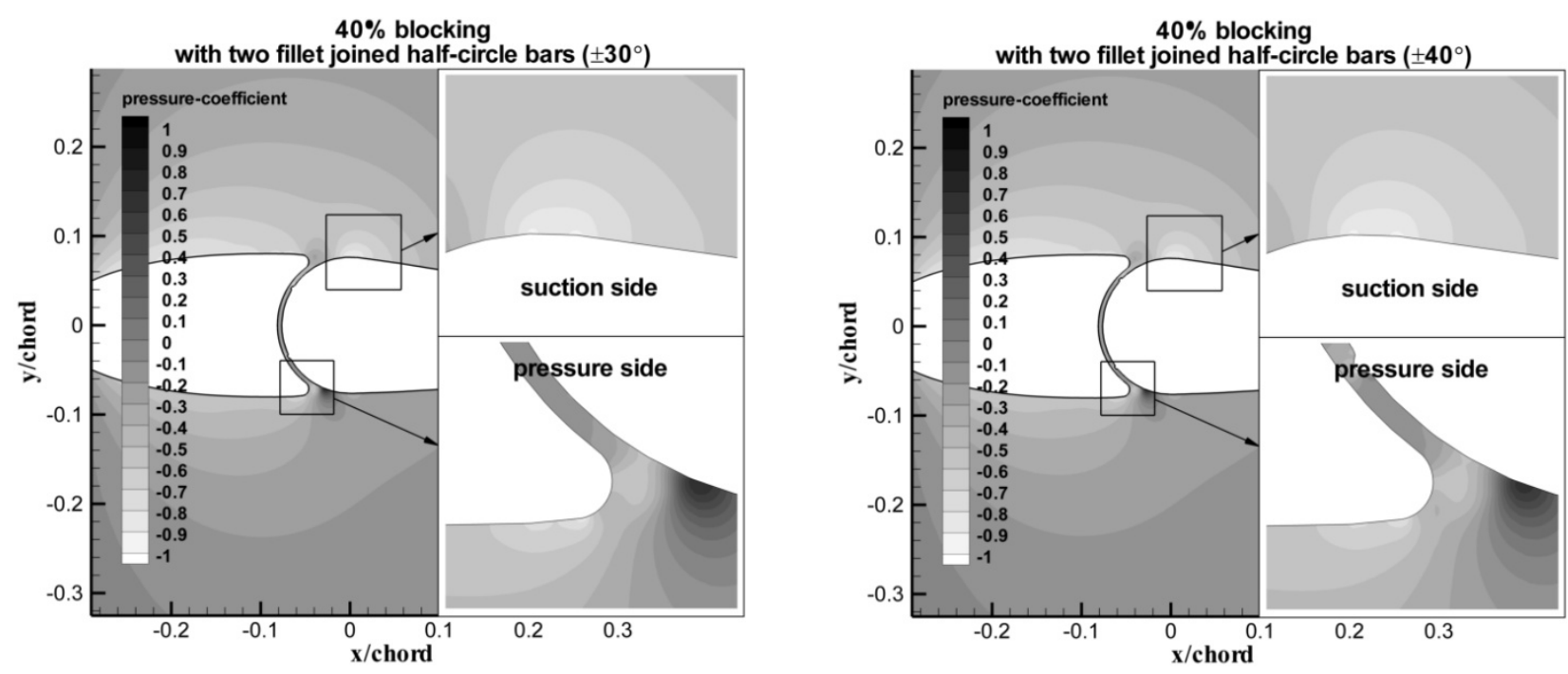

Fig. 10 Pressure distributions around the horn section with fillet jointed half-circular cylindrical bars symmetrically attached at $\pm 30^{\circ}$ (left) and $\pm 40^{\circ}$ (right) with respect to the centre line $\left(R e=4.5 \times 10^{6}\right.$, rudder angle $=3^{\circ}$, flow speed $\left.=6 \mathrm{~m} / \mathrm{s}\right)$.
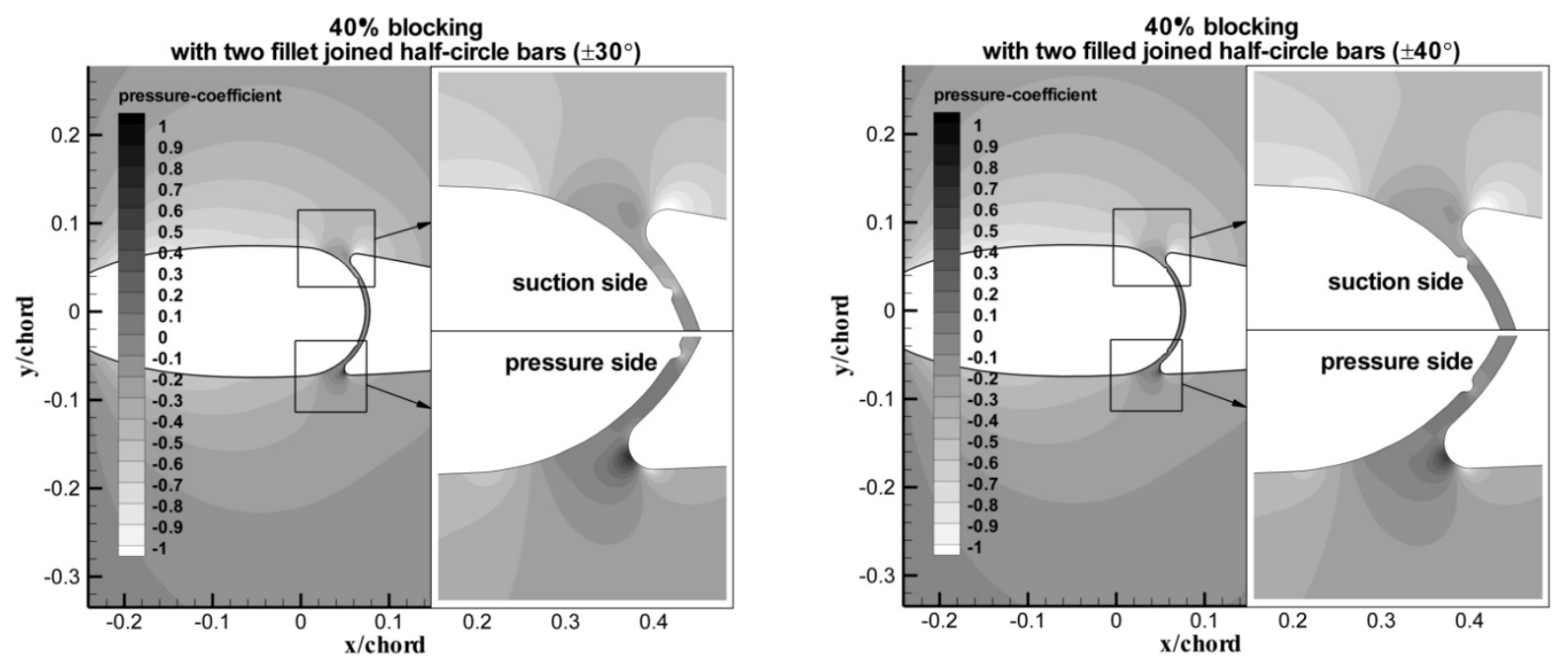

Fig. 11 Pressure distributions around the pintle section with fillet jointed half-circular cylindrical bars symmetrically attached at $\pm 30^{\circ}$ (left) and $\pm 40^{\circ}$ (right) with respect to the centre line $\left(R e=4.5 \times 10^{6}\right.$, rudder angle $=3^{\circ}$, flow speed $\left.=6 \mathrm{~m} / \mathrm{s}\right)$.

\section{The effect of Reynolds number}

Numerical simulations were carried out to investigate the influence of Reynolds number $\left(R_{n}\right)$. The Reynolds number of full scale is $9.6 \times 10^{7}$ of chord-based Reynolds number, $R_{n}=V_{\infty} \cdot C / \mathrm{v}$, where $\mathrm{v}$ is the kinematic viscosity, $C$ is the chord length $(0.746 \mathrm{~m})$, and $V_{\infty}(25 \mathrm{kts})$ is the incoming velocity. Table 6 summarizes the computed results of the gap flow rate with the employment of the symmetric bars attached at $\pm 40^{\circ}$ and without bar at the $R_{n}$ of $9.6 \times 10^{7}$ which is the range of full scale. At the horn section, reduction rates of gap flow with symmetric bars is $43.9 \%$ at the $R_{n}$ of the $9.6 \times 10^{7}$ and the $C_{p}$ has also been also 
increased. Fig. 12 compares the pressure distributions and streamlines near the gap with and without symmetric blocking bars at the full scale Reynolds number. Fig. 12 shows that the $C_{p}$ near the suction side of the movable part changes from -2.171 to 1.540 due to the blocking bars and the streamlines changes in the gap. According to Table 6 and Fig. 12, it can be concluded that the symmetric blocking bars are also effective to reduce gap flow at the full scale Reynolds number.

Table 6 Effect of the gap flow blocking bars symmetrically installed at full scale Reynolds number of $9.6 \times 10^{7}$ (horn section, rudder angle $=3^{\circ}$ ).

\begin{tabular}{|c|c|c|c|}
\hline & Flow rate $(\mathrm{kg} / \mathrm{s})$ & Reduction $(\%)$ & Minimum of $C_{p}$ \\
\hline w/o blocking system & 653.0 & - & -2.171 \\
\hline w/ blocking system & 366.5 & 43.9 & -1.540 \\
\hline
\end{tabular}

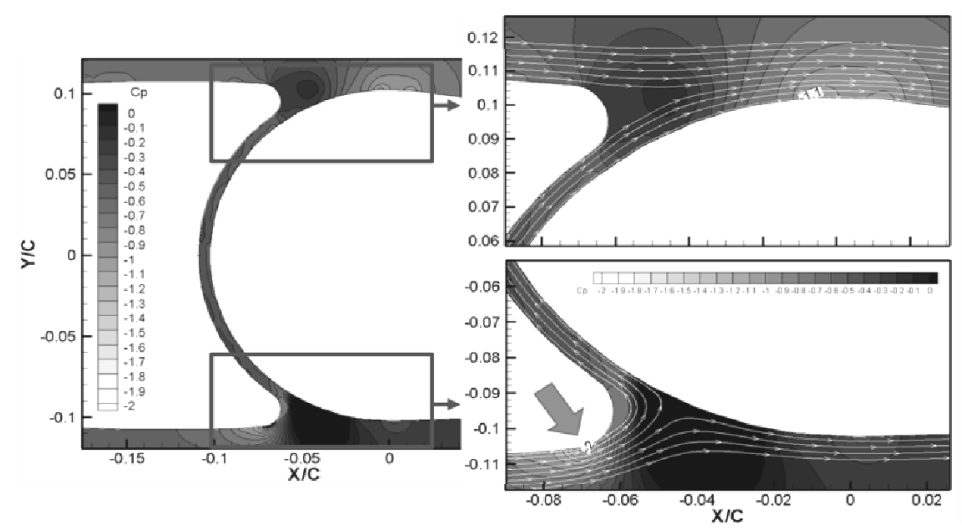

(a) Original horn section.

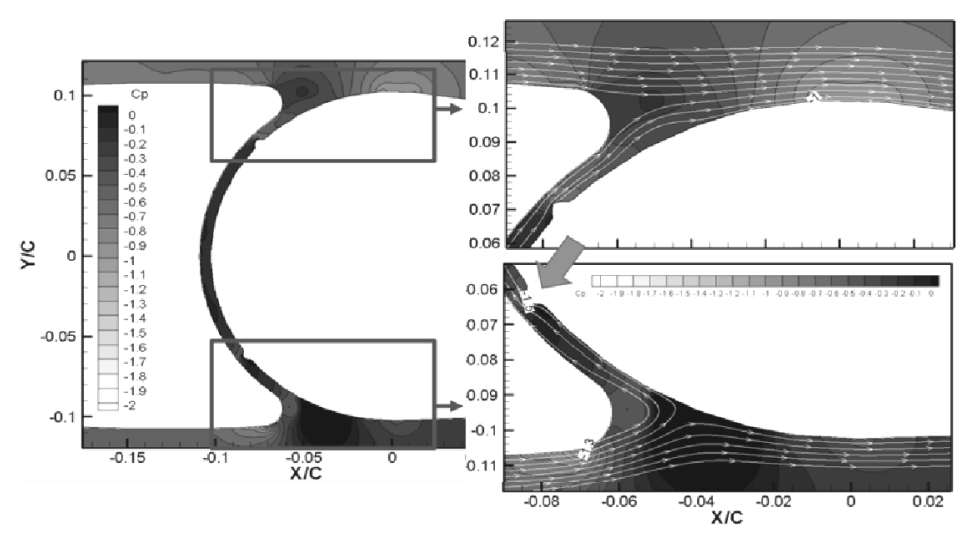

(b) Horn section with symmetric blocking bars.

Fig. 12 Pressure distribution around the horn section with and without symmetric blocking bars at $R n$ of $9.6 \times 10^{7}$ (rudder angle $=3^{\circ}$ ).

\section{Three-dimensional gap flow}

Based on the results of two-dimensional studies, the half circular symmetric bars fillet jointed at the angles of $\pm 40^{\circ}$ with respect to the centerline were chosen as the most adequate blocking device for the three-dimensional horn-type rudder shown in Fig. 1. The symmetric bars are fillet jointed on the movable portion of the horn section and on the fixed portion of the pintle section to maintain simplicity of installment. Numerical computations were carried out at the rudder angle of $3^{\circ}$, as in twodimensional computations, to confirm the capability of the bars in blocking the gap flow. Numerical computations were performed at cavitation number $\sigma=\left(P_{\infty}-P_{v}\right) /\left(0.5 \cdot \rho \cdot U_{\infty}{ }^{2}\right)=1.0$ and operating pressure and vapor pressure of $20307 P a$, and $2340 P a$ respectively. The cavitation model of FLUENT ver. 6.3 is based on the full cavitation model developed by Singhal, Athavale, Li and Jiang (2002). 
Fig. 13 compares the cavitation shape of $\sigma=1.0$ with the void fraction of 0.1 and cavitation shape around the gap and rudder surface with and without the symmetric bars, respectively, and the blocking effects are summarized in Table 7 . The cavitation on horn part of rudder without blocking bar was not existed at $\sigma=1.0$, but the cavitation occurred near the pintle. It can be concluded from the figures that the symmetric bars installed in the three-dimensional horn type rudder can retard the gap flow effectively resulting in a decrease of the low pressure region near the gap. There is no evident cavitation around the blocking bars but cavitation is noticeably increased at the upper corner of the pintle. Although vertical gap flow was blocked by symmetric bars, horizontal gap flow still exists and it can be surmised that the flow spurted from this horizontal gap is much higher. Fig. 13 shows the velocity which indicated on the streamline by the color, was increased and consequently the cavitation near the upper corner of pintle grew larger. Therefore we have to block vertical and horizontal gap simultaneously to reduce cavitation near the gap of rudder

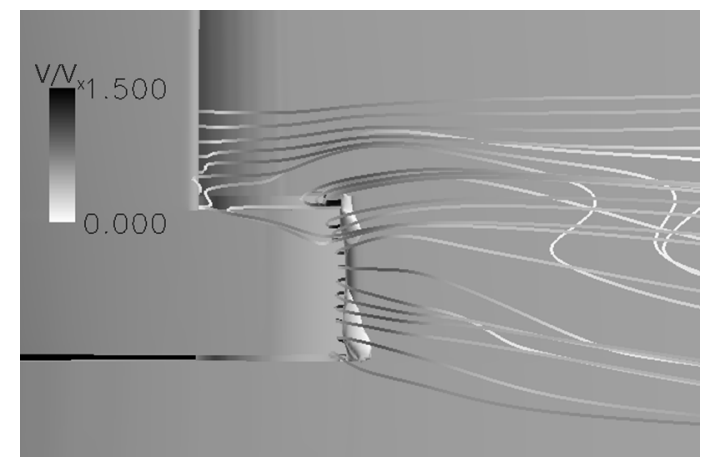

Without blocking bar

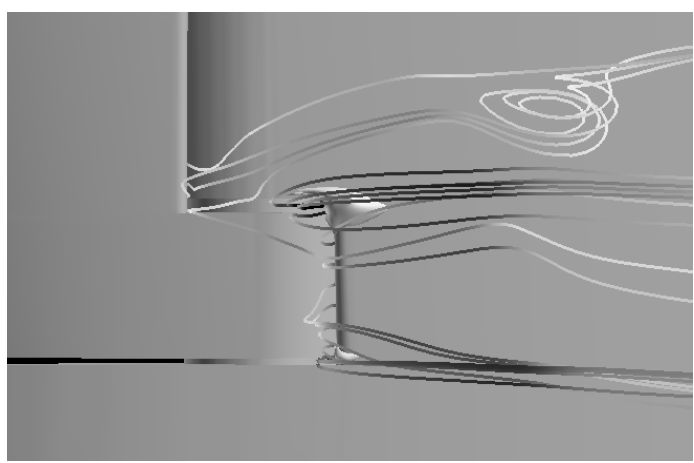

With blocking bars

(a) Streamlines with velocity contour and cavitation shapes on the suction side of the rudder.

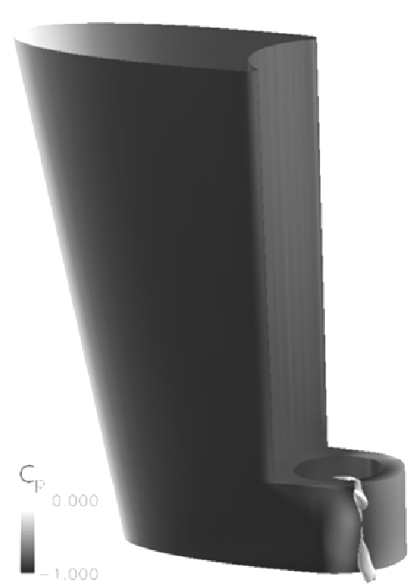

Without blocking bar

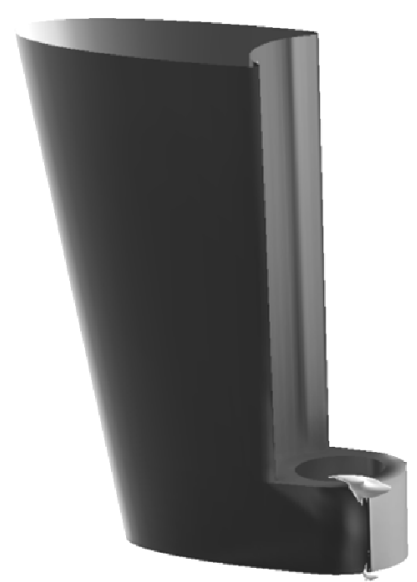

With blocking bars

(b) Pressure distribution on the fixed portion and the blocking bars.

Fig. 13 Cavitation shapes on the suction side and pressure distribution on the fixed portion $\left(\operatorname{Re}=4.5 \times 10^{6}\right.$, rudder angle $=3^{\circ}, \sigma=1.0$, with symmetric blocking bars on horn and pintle sections).

Table 7 Effect of the symmetric bars installed in the three-dimensional rudder model

$\left(R e=4.5 \times 10^{6}\right.$, rudder angle $=3^{\circ}, \sigma=1.0$, with symmetric blocking bars on horn and pintle sections $)$.

\begin{tabular}{|c|c|c|}
\hline & Flow rate $(\mathrm{kg} / \mathrm{s})$ & Reduction $(\%)$ \\
\hline Without blocking bar & 12.5 & - \\
\hline With blocking bar & 7.8 & 37.6 \\
\hline
\end{tabular}




\section{CONCLUSIONS}

The gap flow between the fixed and movable portion of a rudder system is known to be major cause of the rudder cavitation which encounters frequently when a large ship sails at high-speed frequently encounters. As a practical remedy to diminish the rudder gap cavitation, a fillet jointed half-circular cylindrical bar was installed on the surface of the fixed portion facing the gap along the centerplane, but the performance was not satisfying and needs more improvement to substantially retard the gap flow sufficient for the avoidance of rudder cavitation.

In the present study, it was shown through preliminary investigations on a two-dimensional rudder that sectional shapes and the heights of the bars are the most important factors to be considered for efficient blocking of the gap flow. A blocking bar with a rectangular section showed the best performance in blocking the gap flow among tested and the higher protrusion showed the better result. Furthermore, it was found that the conventional fillet jointed half-circular cylindrical bar can also exhibit performance comparable to a single rectangular section if a pair of the bars are installed symmetric to the centerplane.

A pair of half-circular cylindrical bars fillet jointed symmetrically to the centerplane was chosen as the best pick for a gap flow blocking device and was attached to a practical three-dimensional horn type rudder to demonstrate its usefulness in blocking the gap flow and the consequential rudder cavitation. The symmetric blocking bars are convenient to install and maintain and can be mounted easily on the rudder of a ship already in operation. Numerical computations show that the device can reduce the gap flow as much as $41.5 \%$ if compared to those without the blocking device. However, it is also found that cavitation near the upper corner of the pintle becomes more intense even when the gap flow has been successfully retarded with the blocking bars. It can be speculated that the phenomenon may arise from the increase of the flow through the horizontal break and hence the influence of the flow through the horizontal breaks of a horn type rudder on the cavitation behavior needs to be studied in near future.

\section{ACKNOWLEDGEMENTS}

This work was supported by the Inha University and the National Research Foundation Grant(2007-0055774, 20110026463).

\section{REFERENCES}

Boo, G.T., Song, I.H. and Shin, S.C., 2004. Numerical Simulation for the Rudder in order to Control the Cavitation Phenomena. Journal of Ship and Ocean Technology, 8(1), pp.42-50.

Kim, M.C., Lee, U.S. and Byun, T.Y., 2008. Study on Optimization of Anti-erosion Rudder Section of Large Container Ship by Genetic Algorithm. Journal of the Society of Naval Architects of Korea, 45(3), pp.403-410.

Paik, B.G., Kim, K.Y., Ahn, J.W., Kim, Y.S., Kim, S.P. and Park, J.J., 2008. Experimental study on the gap entrance profile affecting rudder gap cavitation. Ocean Engineering, 35(1), pp.139-149.

Park, K.R and Lee, Y.G., 2010. A Study on the Rudder Shapes for the Suppression of Cavitation around a Horn-type Rudder. Journal of the Society of Naval Architects of Korea, 47(4), pp.553-564.

Rhee, S.H., Lee, C.M., Lee, H.B. and Oh, J., 2010. Rudder Gap Cavitation: Fundamental Understanding and Its Suppression Devices. International Journal of Heat and Fluid Flow, 31(4), pp.640-650.

Rhee, S.H. and Kim, H., 2008. A Suggestion of Gap Flow Control Devices for the Suppression of Rudder Cavitation. Journal of Marine Science and Technology, 13(4), pp. 356-370.

Singhal, A.K., Athavale, M.M., Li, H. and Jiang, Y., 2002. Mathematical bases and validation of the full cavitation model. Journal of Fluid Engineering, 124(3), pp.167-264.

Shen, Y.T., Jiang, C.W. and Remmers, K.D., 1997. A Twisted Rudder for Reduced Cavitation. J. Ship Research, 41(4), pp. 260-272.

Seo, D.W., Lee, S.H., Oh, J.K. and Kim, H., 2010. A Numerical Study for the Efficacy of Flow Injection on the Diminution of Rudder Cavitation. International Journal of Naval Architecture and Ocean Engineering, 2(2), pp.104-111.

Seo, D.W., Lee, S.H., Kim. S.H. and Oh, J.K., 2012. Practically applicable devices for blocking the gap flow of a horn rudder to reduce rudder cavitation and their verification through numerical simulations. J Mar Sci Technol, 17(1), pp.18-29.

Guo, B., Langrish, T.A.G. and Fletcher, D.F., 2001. Simulation of turbulent swirl flow in an axisymmetric sudden expansion. AIAA journal, 39(1). pp.96-102. 